3 Ultrasound-Mediated Long-Circulating

4 Nanopolymer Delivery of Therapeutic siRNA and

5 Antisense MicroRNAs Leads to Enhanced

6 Paclitaxel Sensitivity in Epithelial Ovarian Cancer

\title{
7 Chemotherapy
}

8

Yi Liu ${ }^{1,2}$, Tengfei Long ${ }^{1,2}$, Ni Zhang ${ }^{3}$, Bin Qiao², Yang Qiang ${ }^{2,4}$, Yuanli Luo², Jin Cao², Jing Luo $^{5}$, Dong Yuan ${ }^{1}$, Yixuan Sun ${ }^{1}$, Yanxi Li ${ }^{6}$, Zhu Yang ${ }^{1, *}$, ZG Wang,"*

${ }^{1}$ Department of Gynecology and Obstetrics, the Second Affiliated Hospital of Chongqing Medical University, Chongqing 400010, China;

${ }^{2}$ Institute of Ultrasound Imaging, the Second Affiliated Hospital of Chongqing Medical University, Chongqing 400010, China;

${ }^{3}$ Department of Oncology, the Second Affiliated Hospital of Chongqing Medical University Chongqing 400010, China;

${ }^{4}$ Department of Neurosurgery, the Second Affiliated Hospital of Chongqing Medical University Chongqing 400010, China;

${ }^{5}$ Department of Pathology, Chongqing Medical University, Chongqing 400010, China; ${ }^{6}$ Department of Reproductive Medicine Center, Daping Hospital, Army Medical University, Chongqing 400042, China;

*Corresponding authors: 
35

\begin{tabular}{|c|c|c|c|c|}
\hline & Size $(\mathrm{nm})$ & $\begin{array}{c}\text { Zeta } \\
\text { Potential }(\mathrm{mV})\end{array}$ & $\begin{array}{c}\text { Encapsulation } \\
\text { Efficiency (\%) } \\
\text { (siRNA / anti-miR) }\end{array}$ & $\begin{array}{l}\text { Polydispersity } \\
\text { Index }\end{array}$ \\
\hline NC-NPs & $139.52 \pm 18$ & $3.55 \pm 3$ & - & 0.008 \\
\hline LPHNPs & $151.07 \pm 19$ & $7.3 \pm 2$ & $81.18 \pm \underset{3}{5 / 80.65 \pm}$ & 0.121 \\
\hline FaLPHNPs & $169.25 \pm 21$ & $5.28 \pm 3$ & $\begin{array}{c}82.33 \pm 4 / 81.27 \pm \\
7\end{array}$ & 0.09 \\
\hline
\end{tabular}

36

37

38

39

40

41
Zhu Yang, E-mail addresses: yangzhu@hospital.cqmu.edu.cn

ZG Wang, E-mail addresses: 303507@hospital.cqmu.edu.cn

\section{Supplementary Figures}

Table S1 Physical characteristics of non-RNAs-loaded nanopolymers (NC-NPs), nontargeted lipid-PLGA hybrid nanopolymers (LPHNPs) and targeted lipid-PLGA hybrid nanopolymers (FaLPHNPs). 
PLGA
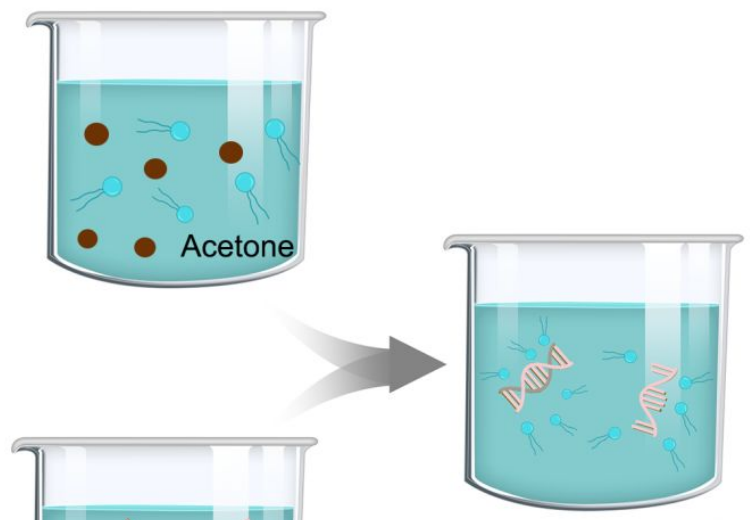

Lecithin
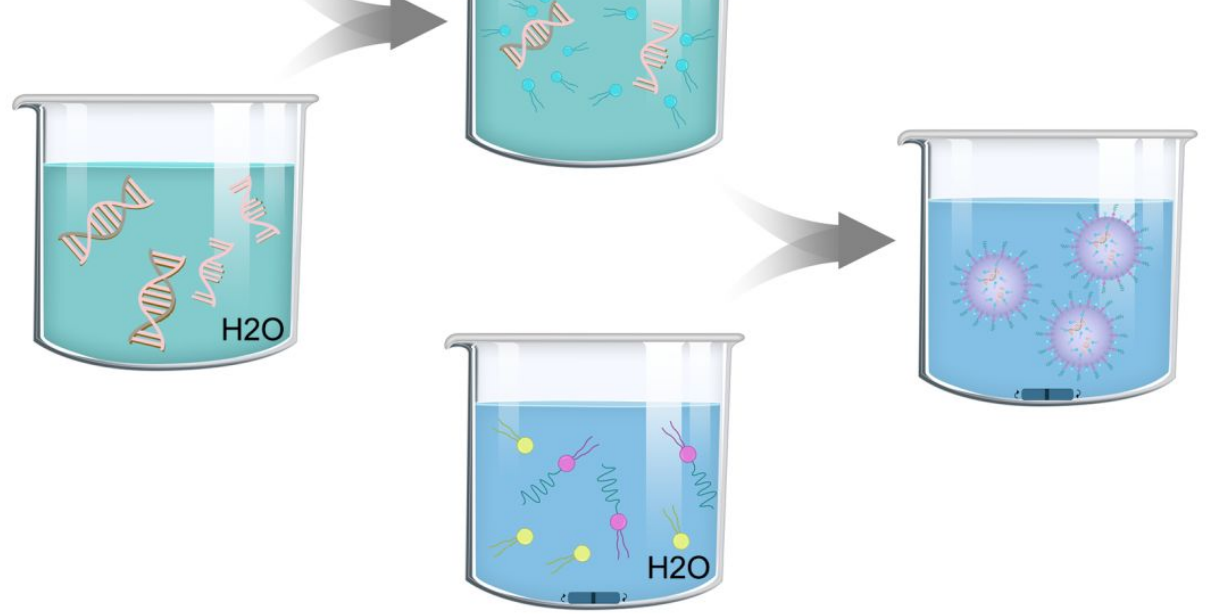

DSPE-PEG5k-Folic acid

DOTAP-Chol

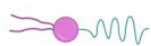

SIK2 SiRNA

Anti-miR21

45 Figure S1 Flow chart of nanopolymer synthesis. PLGA and DOTAP-Chol were dissolved in

46 the acetone solution; SIK2 siRNA and anti-miR21 solution are mixed into acetone

47 suspension to form siSIK2/anti-miR21/DOTAP-Chol nanocomplexes. Then, the polyplex

48 solution with the nanopolymer was added into the Milli-Q water containing lecithin and

49 DSPE-PEG5K-Fa. The nanopolymers were formed immediately upon blending. Next, the 50 above solution was stirred for 2 4 hours to evaporate residual acetone. 


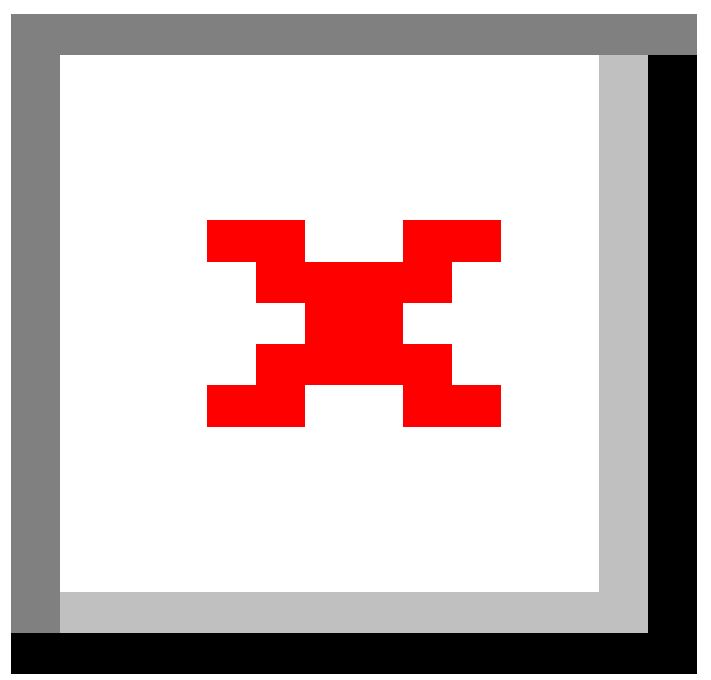

Figure S2. Evaluation of the cell uptake capacity of NPs. (a) LSCM images of SKOv3 cells treated with FaLPHNPs, with or without US-MB mediation, and non-targeted NPs (LPHNPs)

$591 \mathrm{~h}$ post-treatment. The blue fluorescence (DAPI) represents nuclei of SKOv3 cells, the red 60 fluorescence (Cy3) reveals Cy3-labeled SIK2 siRNA (scale bar $=20 \mu \mathrm{m})$. (b) Flow

61 cytometry analysis with LSCM was used for evaluating the capacity of targeted delivery of 62 various NPs in vitro at the same time point. (c) Transfection rate analysis of LPHNPs, 63 FaLPHNPs, and FaLPHNPs + US-MB after incubation with SKOv3 cell for $1 \mathrm{~h}$. 


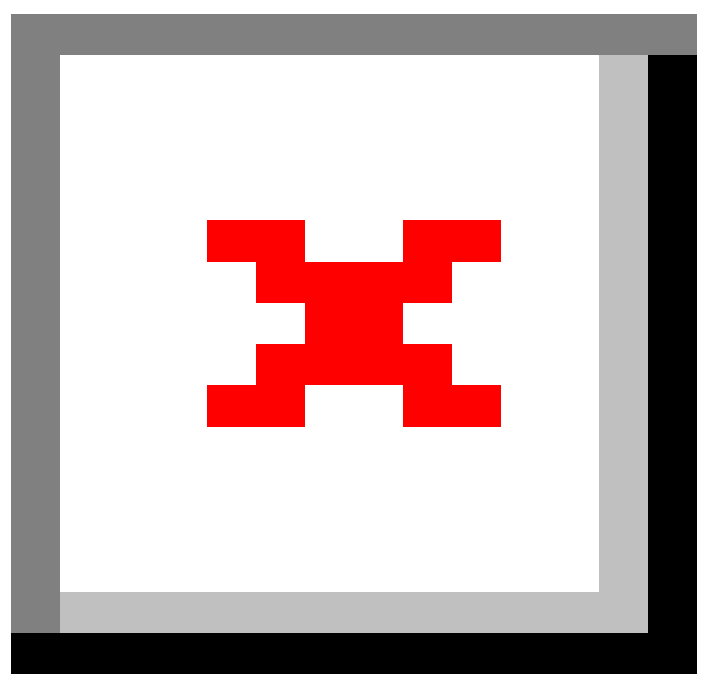

67 Figure S3. Evaluation of the cell uptake capacity of NPs. (a) LSCM images of A2780 cells 68 treated with FaLPHNPs, with or without US-MB mediation, and non-targeted NPs (LPHNPs)

$691 \mathrm{~h}$ post-treatment. The blue fluorescence (DAPI) represents nuclei of A2780 cells, the red 70 fluorescence $(\mathrm{Cy} 3)$ reveals Cy3-labeled SIK2 siRNA (scale bar $=20 \mu \mathrm{m})$. (b) Flow

71 cytometry analysis with LSCM was used for evaluating the capacity of targeted delivery of 72 various NPs in vitro at the same time point. (c) Transfection rate analysis of LPHNPs, 73 FaLPHNPs, and FaLPHNPs + US-MB after incubation with A2780 cell for $1 \mathrm{~h}$. 
a

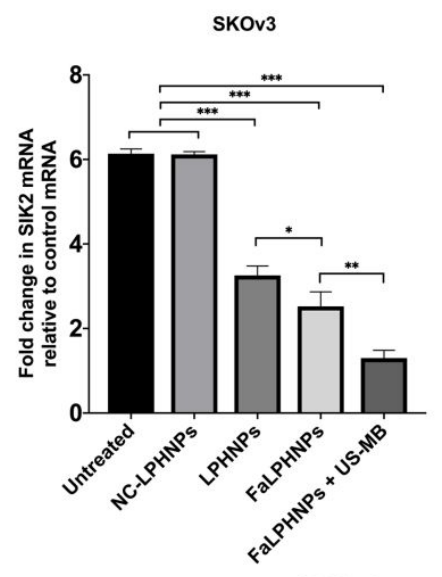

d

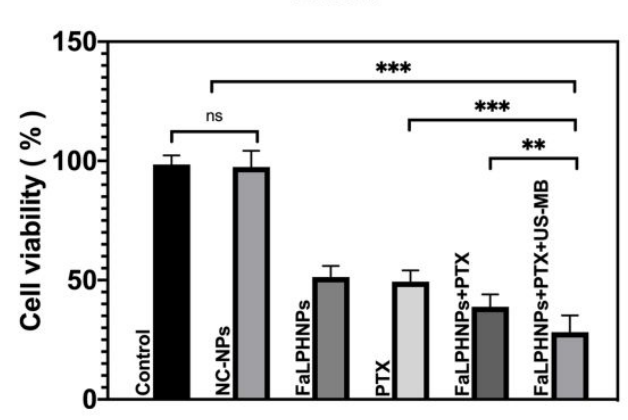

C

SKOv3

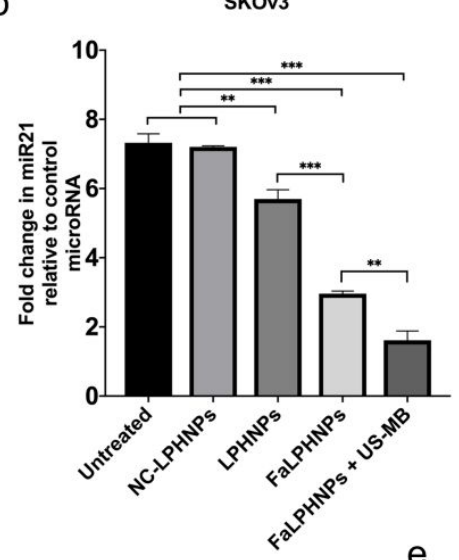

e

FaLPHNP +

Untreated LPHNPS FaLPHNPS US-MB

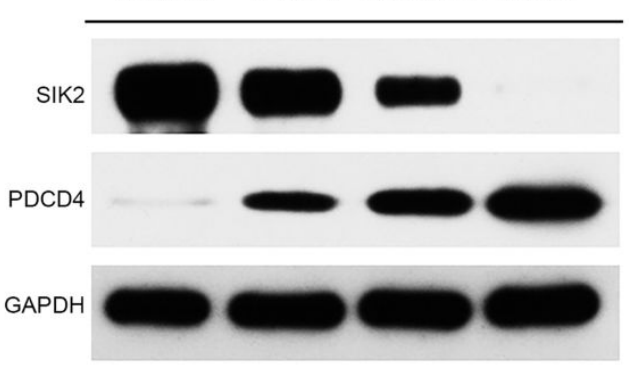

Figure S4. (a) Quantification of endogenous SIK2 mRNA expression levels in SKOv3 cells and in SKOv3 cells $48 \mathrm{~h}$ post-transfection; $n=3$ each. (b) Quantification of endogenous miR21 expression levels in SKOv3 cells and in SKOv3 cells $48 \mathrm{~h}$ post-transfection. (c) Cytotoxicity of SKOv3 cells incubated with different concentrations of SIK2 siRNA encapsulated in FaLPHNPs for $48 \mathrm{~h}$ with or without anti-miR21 treatment. (d) Viability of cells treated with complementary FaLPHNPs along with $50 \mathrm{nM}$ siSIK2 and $100 \mathrm{nM}$ antimiR2 1 and US-MB. (e) Western blotting assay of SIK2 and PDCD4 after treatment with LPHNPs (siSIK2/anti-mi21), FaLPHNPs (siSIK2/anti-mi21) and FaLPHNPs (siSIK2/antimi21) + US-MB, with GAPDH as control. 


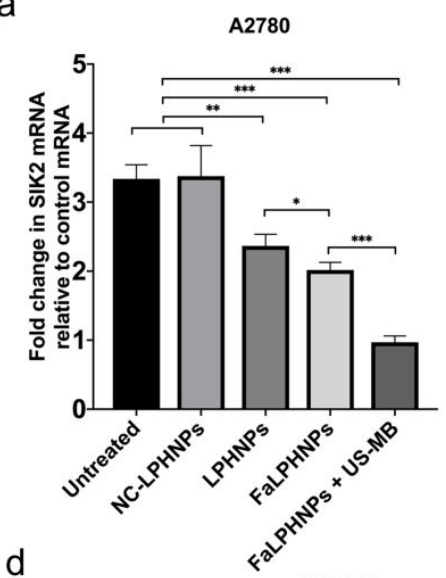

d

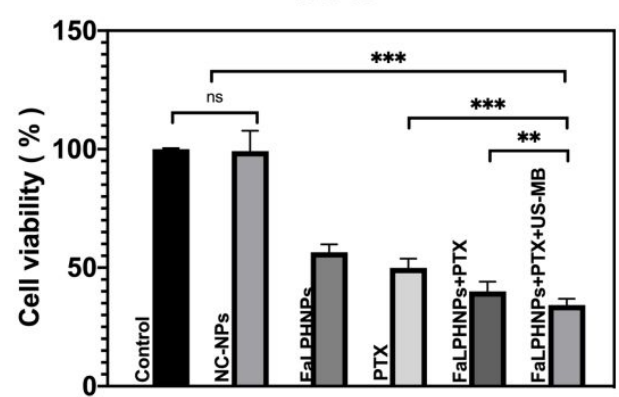

b

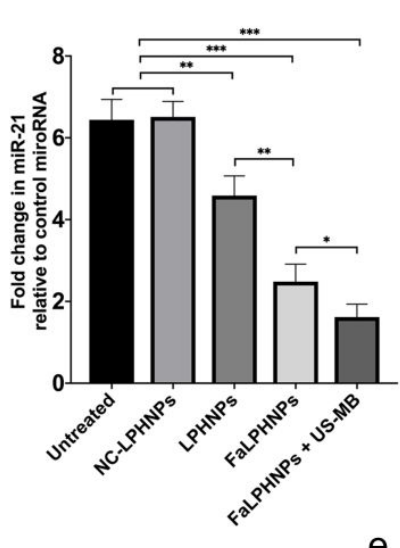

e

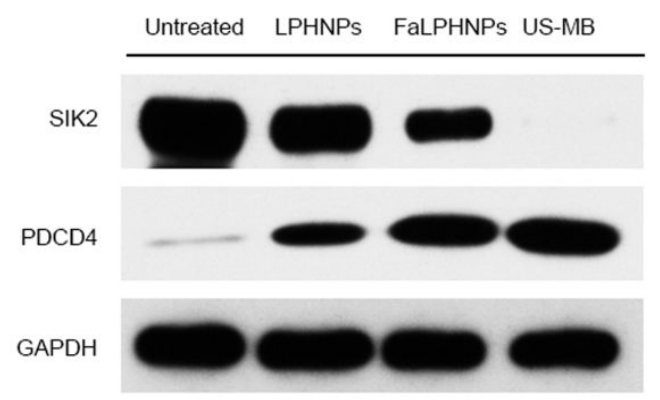

Figure S5. (a) Quantification of endogenous SIK2 mRNA expression levels in A2780 cells and in A2780 cells $48 \mathrm{~h}$ post-transfection; $\mathrm{n}=3$ each. (b) Quantification of endogenous miR21 expression levels in A2780 cells and in A2780 cells $48 \mathrm{~h}$ post-transfection. (c) Cytotoxicity of A2780 cells incubated with different concentrations of SIK2 siRNA encapsulated in FaLPHNPs for $48 \mathrm{~h}$ with or without anti-miR21 treatment. (d) Viability of cells treated with complementary FaLPHNPs along with $50 \mathrm{nM}$ siSIK2 and $100 \mathrm{nM}$ antimiR2 1 and US-MB. (e) Western blotting assay of SIK2 and PDCD4 after treatment with LPHNPs (siSIK2/anti-mi21), FaLPHNPs (siSIK2/anti-mi21) and FaLPHNPs (siSIK2/antimi21) + US-MB, with GAPDH as control. 

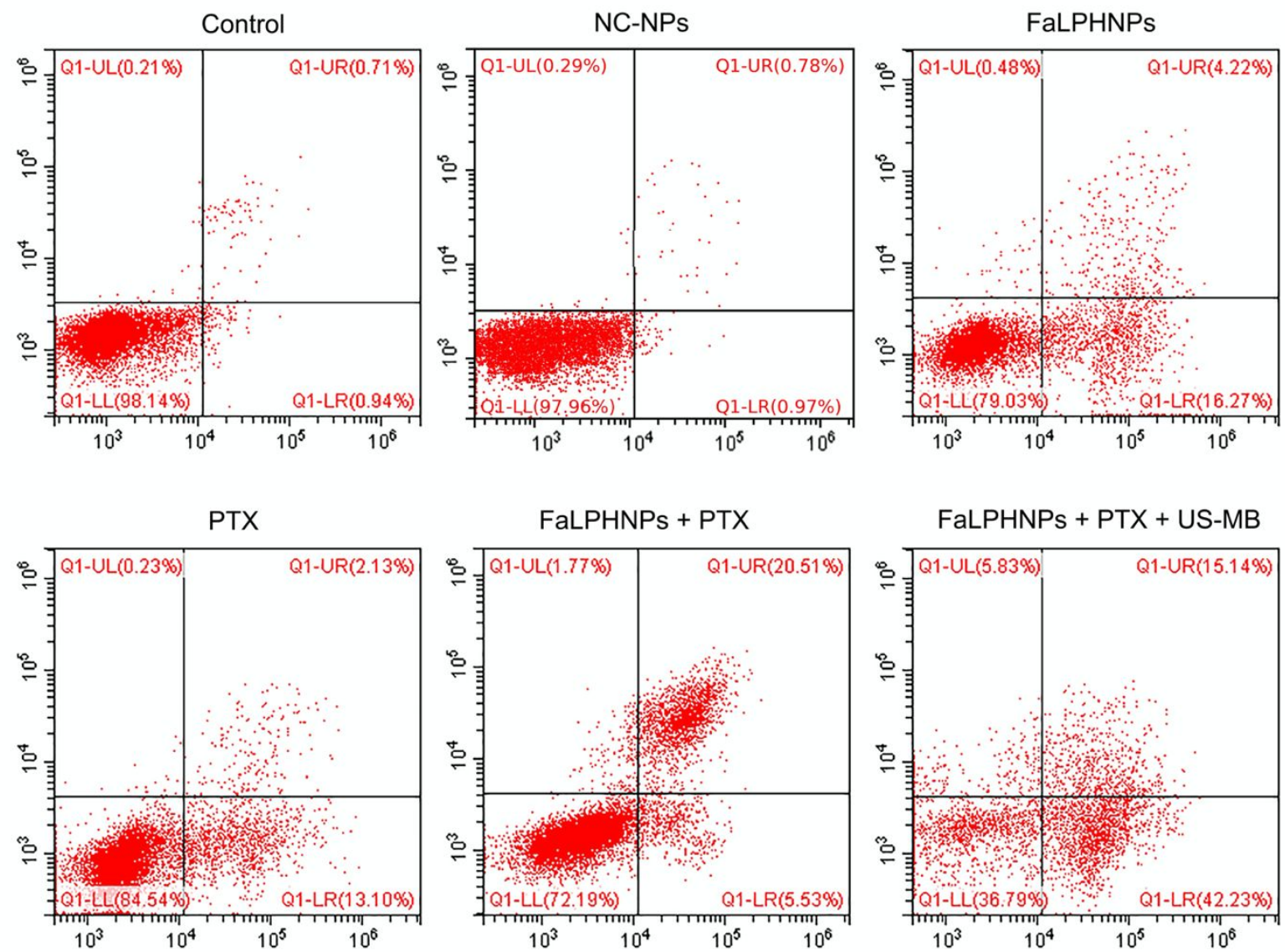

Figure S6. Cell apoptosis in OVCAR3 cells 48h post-treatments. The X-axis represents the amount of phosphatidylserine (PS)-conjugated Annexin V, and the Y-axis for DAPI.

a

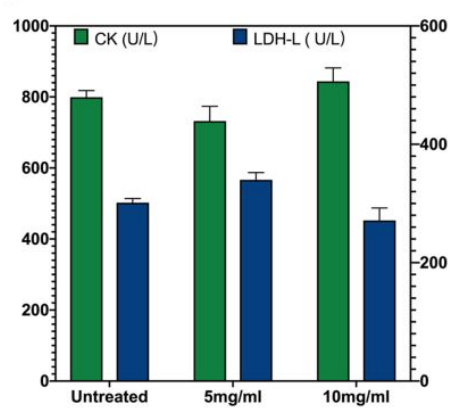

b

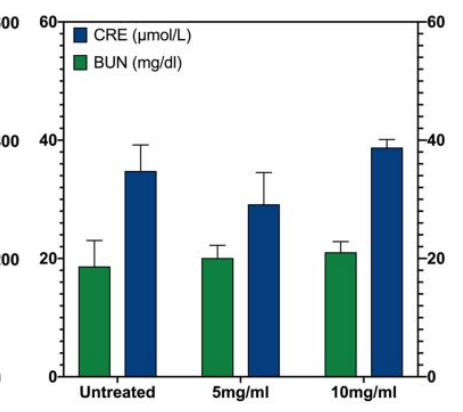

C

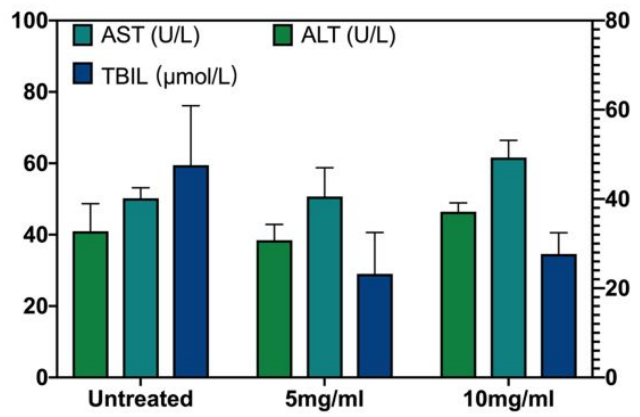

Figure S7. Blood biochemical analysis. (a) CK and LDH-L represent for heart function, (b) function. Blood was withdrawn 7-days after injection of different concentrations of FaLPHNPs(siSIK2/anti-miR21) from mice. 